\title{
The significance of LRPPRC overexpression in gastric cancer
}

\author{
Xiaosa Li $\cdot$ Lifen Lv $\cdot$ Jianyong Zheng $\cdot$ Jinfeng Zhou \\ Bing Liu $\cdot$ Hui Chen $\cdot$ Cong Liang $\cdot$ Rui Wang $\cdot$ Linna Su \\ Xiaohua Li $\cdot$ Daiming Fan
}

Received: 27 November 2013/Accepted: 6 December 2013/Published online: 28 December 2013

(C) The Author(s) 2013. This article is published with open access at Springerlink.com

\begin{abstract}
LRPPRC is a multifunctional protein involved in mitochondrial gene expression and function, cell cycle progression, and tumorigenesis. We analyzed LRPPRC gene expression in 253 paired cases of gastric cancer and noncancerous regions and six gastric cancer cell lines to demonstrate the importance of LRPPRC expression for the prediction of prognosis of gastric cancer. Our results showed that LRPPRC expression in gastric cancer tissues is significantly higher than that in paired control tissue $(P<0.001)$. Patients with higher LRPPRC expression showed a poorer overall survival rate than those with lower LRPPRC expression $(P<0.001)$. Multivariate analysis demonstrated that lymph node metastasis $(\mathrm{N})$, distant metastasis (M), TNM stage, and LRPPRC expression were independent prognostic factors for gastric cancer $(P=0.004,0.002,0.017,0.004$ respectively).Moreover, Western blotting showed that LRPPRC expression was increased in SGC7901, BGC823, MKN45, and XGC9811cells. The in vitro proliferation assay showed that LRPPRC expression is inversely associated with gastric cancer cells growth. Our results indicated that LRPPRC could be used as a predictive marker for patient prognosis
\end{abstract}

Xiaosa Li, Lifen Lv and Jianyong Zheng have contributed equally to this study, and all should be considered first author.

X. Li · L. Lv · J. Zheng · J. Zhou · B. Liu · H. Chen ·

C. Liang $\cdot$ R. Wang $\cdot$ L. Su $\cdot$ X. Li $(\bowtie) \cdot$ D. Fan $(\bowtie)$

State Key Laboratory of Cancer Biology, Xijing Hospital of

Digestive Diseases, Fourth Military Medical University, 127\#

Changle West Road, Xi'an 710032, China

e-mail: fmmulxh@yahoo.com.cn

D. Fan

e-mail: fandaim@fmmu.edu.cn of gastric cancer and may be a novel therapeutic target for gastric cancer in future.

Keywords LRPPRC - LRP130 - Gastric cancer · Expression · Prognosis

\section{Introduction}

Gastric cancer is a disease with one of the poorest prognoses, being the second cause of tumor-related mortality in the world. Five-year overall survival is $25 \%$ or less, especially in USA, Europe, and China [1, 2]. Every year, 1 million new cases of gastric cancer are diagnosed and 700,000 die of this disease worldwide [3, 4].Most patients with gastric cancer are diagnosed with advanced gastric cancer, and overall survival rate remains poor. To provide new insights into the pathology of the disease and to permit earlier diagnosis, there is a need for new prognostic tumor markers that are more sensitive than those currently available, such as CEA and CA19-9 [5].

In 2000, Small and Peeters [6] described a set of proteins with 35 amino acid repeat sequences that were dubbed 'pentatricopeptide repeat cassette proteins'. Members of the pentatricopeptide repeat (PPR) protein family play important roles in mitochondrial RNA metabolism in metazoans, plants, and yeast [7]. LRPPRC protein (also known as LRP130) [8, 9], a member of PPR protein family, regulates the stability and handling of mature mitochondrial mRNA and participates in the formation of the transcriptional activator PGC-1 involved in liver glucose homeostasis, energy metabolism, and nuclear receptor activation [10].

Previous reports have showed that LRPPRC was highly expressed in most cancers, such as hepatoma cancer, lung adenocarcinoma, esophageal squamous cell carcinoma, 
colon cancer and lymphoma, and significantly associated tumorigenesis and invasion [11]. However, the LRPPRC expression in gastric cancer and its correlation with gastric cancer clinicopathological characteristics is still unclear.

In this study, we first examined LRPPRC expression in 253 paired cases of gastric cancer and paired noncancerous regions and six gastric cancer cell lines to investigate the relevance of LRPPRC expression and its relation to clinicopathological characteristics. Besides, an in vitro study was performed to observe the LRPPRC effect on gastric cancer cell proliferation. In conclusion, our results suggested that LRPPRC is a novel independent marker for the prognosis with functional relevance in gastric cancer.

\section{Materials and methods}

Clinical tissue samples

Our study included 253 patients (153 males, 100 females; mean age 65.5 years; range 34-83), who underwent surgery at Xijing Hospital, Fourth Military Medical University (Xi' an, China), were recruited between May 2003 and August 2005 after obtaining their written informed consent. Cancer tissues, along with normal tissues that were at least $5 \mathrm{~cm}$ away from the cancer, were obtained from the patients. The median follow-up period for survivors was 47 months (range 0-128 months) by telephone and mail. The study items included age, gender, location of the tumor, tumor stage, depth of invasion, lymph node metastasis, distant metastasis, and tumor-node-metastasis (TNM) stage. Patient characteristics are summarized in Table 1. All of the patients were staged using the 7th edition of the International Union Against Cancer TNM staging system. Of the 253 patients, 66(26\%) had T1-stage, 60(24\%) had T2-stage, 84(33\%) had T3-stage, and 43(17\%) had T4-stage gastric cancer. Tissues were fixed in $10 \%$ formaldehyde, embedded in paraffin, cut into $4-\mu \mathrm{m}$ sections, and mounted on slides.

\section{Immunohistochemical staining}

The cancer and noncancerous tissues from 253 patients were embedded in paraffin and cut into sections for immunohistochemical analysis. Slides were baked at $60{ }^{\circ} \mathrm{C}$ for $2 \mathrm{~h}$, followed by deparaffinization with xylene, and rehydrated, after being washed three times in PBS (phosphate-buffered saline), then using a pressure cooker with $10 \mathrm{nM}$ citrate buffer (PH 6.0) for 5 min. After rinsing with PBS. They were then treated with $3 \%$ hydrogen peroxide for $12 \mathrm{~min}$ in methanol to quench endogenous peroxidase activity, followed by incubation with $1 \%$ bovine serum albumin to block nonspecific binding for $1 \mathrm{~h}$. The antigen-antibody reaction was carried out overnight at $4{ }^{\circ} \mathrm{C}$ with the anti-
Table 1 Correlation between clinicopathological characteristics and LRPPRC expression

\begin{tabular}{|c|c|c|c|c|c|}
\hline Total & $n$ & $\begin{array}{l}\text { LRPPRC } \\
( \pm)\end{array}$ & $\begin{array}{l}\text { LRPPRC } \\
(++)\end{array}$ & $\begin{array}{l}\text { LRPPRC } \\
(+++)\end{array}$ & $P$ \\
\hline Age & & & & & $0.617^{\mathrm{a}}$ \\
\hline$\leq 65.5$ & 131 & 41 & 48 & 42 & \\
\hline$>65.5$ & 122 & 40 & 35 & 47 & \\
\hline Gender & & & & & $0.357^{\mathrm{a}}$ \\
\hline Male & 153 & 51 & 52 & 50 & \\
\hline Female & 100 & 30 & 31 & 39 & \\
\hline Location & & & & & $0.905^{\mathrm{b}}$ \\
\hline Gastric antrum & 105 & 39 & 26 & 40 & \\
\hline Lessercurvature & 75 & 23 & 27 & 25 & \\
\hline Gastric cardia & 73 & 19 & 30 & 24 & \\
\hline $\mathrm{T}$ & & & & & $<0.001^{\mathrm{b}}$ \\
\hline $\mathrm{T} 1$ & 66 & 42 & 17 & 7 & \\
\hline $\mathrm{T} 2$ & 60 & 23 & 29 & 8 & \\
\hline T3 & 84 & 9 & 27 & 48 & \\
\hline $\mathrm{T} 4$ & 43 & 7 & 10 & 26 & \\
\hline $\mathrm{N}$ & & & & & $<0.001^{\mathrm{b}}$ \\
\hline No & 58 & 32 & 18 & 8 & \\
\hline N1 & 63 & 25 & 27 & 11 & \\
\hline $\mathrm{N} 2$ & 97 & 19 & 29 & 49 & \\
\hline N3 & 35 & 5 & 9 & 21 & \\
\hline $\mathrm{M}$ & & & & & $0.002^{\mathrm{a}}$ \\
\hline M0 & 219 & 76 & 74 & 69 & \\
\hline M1 & 34 & 5 & 9 & 20 & \\
\hline TNM & & & & & $0.880^{\mathrm{b}}$ \\
\hline 1 & 33 & 11 & 13 & 9 & \\
\hline 2 & 92 & 26 & 34 & 32 & \\
\hline 3 & 101 & 35 & 29 & 37 & \\
\hline 4 & 27 & 9 & 7 & 11 & \\
\hline Tumor grade & & & & & $0.748^{\mathrm{b}}$ \\
\hline Well & 34 & 11 & 14 & 9 & \\
\hline Moderately & 138 & 43 & 44 & 51 & \\
\hline Poorly & 81 & 27 & 25 & 29 & \\
\hline
\end{tabular}

\footnotetext{
a $P$ value for expression levels compared by Mann-Whitney test

b $P$ value for expression levels compared by Kruskal-Wallis test
}

LRPPRC antibody diluted 1:500(Santa Cruz Biotechnology Inc., Santa Cruz, CA). Rinsed for three times in PBS and incubated with a horseradish-peroxidase-conjugated antiIgG antibody (1:3,000; Santa Cruz) for $1 \mathrm{~h}$. Finally, the sections were developed with 3,3'-diaminobenzidine solution for $2 \mathrm{~min}$, washed briefly in running water, counterstained with hematoxylin, dehydrated through a graded series of alcohol to xylene and were then mounted with Permount onto coverslips. Images were obtained under a light microscope (Olympus BX51;Olympus, Japan) equipped with a DP70 digital camera. As negative controls, tissue sections were processed under the same experimental 
conditions described above, except that they were incubated overnight at $4{ }^{\circ} \mathrm{C}$ in blocking solution without the antiLRPPRC antibody.

\section{Immunohistochemical analysis}

Staining of LRPPRC was detected mainly in the cytoplasm of tumor cells. The degree of immunostaining was reviewed and scored independently by two pathologists who did not know the clinical features or survival status of the patients then viewed the stained tissue slides separately. An average value of two independent scores was presented in the present study [12-14]. Expression of LRPPRC was evaluated according to the ratio of positive cells per specimen and staining intensity. The ratio of positive cells per specimen was evaluated quantitatively and scored as follows: $0=$ staining of $\leq 1 \% ; 1=$ staining of $2-25 \% ; 2=$ staining of $26-50 \%$; $3=$ staining of $51-75 \%$; and $4=$ staining of $>75 \%$ of the cells examined. Intensity was graded as follows: $0=$ no signal; $1=$ weak; $2=$ moderate; and $3=$ strong. A total score of 0-12 was finally calculated and graded as negative (-; score: $0-1)$, weak $(+$; score: $2-4)$, moderate $(++$; score: 5-8), and strong (+++; score: 9-12) [14, 15].

Cell culture, plasmid construction, and cell transfection

Gastric cancer cell lines (KATOIII, SGC7901, BGC823, MKN45, MKN28, and XGC9811) were maintained in Dulbecco's modified Eagle's medium (Gibco RL, Grand Island, NY) supplemented with $10 \%$ fetal bovine serum, $100 \mathrm{U} / \mathrm{ml}$ penicillin, and $0.1 \mathrm{mg} / \mathrm{ml}$ streptomycin. And incubated at $37{ }^{\circ} \mathrm{C}, 5 \% \mathrm{CO}_{2}$. For the small interference RNA (siRNA)knockdown experiment, double-stranded RNA duplexes that targeted the human LRPPRC gene (5'-CACCGGAGGAG CATTTGAGACAATATTCAAGAGATATTGTCTCAAA TGCTCCTCCTTTTTTG-3' $/ 5^{\prime}$-GATCCAAAAAAGGAGGAGCATTTGAGACAATATCTCTTGAATATTGTCTC AAATGCTCCTCC-3') were synthesized, negative control (NC) siRNA was also synthesized. Gastric cancer cell lines were transfected with siRNA at concentration of $20 \mathrm{lmol} / \mathrm{L}$ with lipofectamine (RNAiMAX, Invitrogen), incubated in glucose-free Opti-MEM (Invitrogen) for the time indicated, and analyzed by the proliferation assay. All siRNA duplexes were used together as a triple transfection. siRNA knockdowns were performed in four Gastric cancer cell lines to evaluate proliferation value under LRPPRC suppression. The values are presented as mean \pm standard deviation (SD) from independent experiments conducted in triplicate.

\section{Western blot}

Cells were washed twice with cold PBS and lysed on ice in RIPA buffer with protease inhibitors and quantified by
BCA method. $50 \mathrm{mg}$ Protein lysates were resolved on $8 \%$ SDS polyacrylamide gel, electrotransferred to polyvinylidene fluoride membranes (Millipore, Bedford, MA) and blocked in $5 \%$ nonfat dry milk in Tris-buffered saline ( $\mathrm{pH}=7.5$ ). Membranes were immunoblotted overnight at $4{ }^{\circ} \mathrm{C}$ with anti-LRPPRC polyclonal antibodies as IHC described above, respectively, then followed by their respective secondary antibodies. Signals were detected by enhanced chemiluminescence (Pierce, Rockford, IL). For Immunofluorescence, the binding of primary antibody was visualized by anti-rabbit IgG antibody, and the slides were then examined by a confocal laser scanning microscope.

\section{Proliferation assays}

In gastric cancer cell lines transfected with siRNA, $1 \times 10^{5}$ cells were seeded in 12-well dishes and cultured for $96 \mathrm{~h}$ to determine proliferation. Viable cells were counted every day by reading the absorbance at $490 \mathrm{~nm}$ using a 96-plate reader BP800 (Dynex Technologies, Chantilly, VA, USA). Each experiment was performed in triplicate.

\section{Statistical analysis}

All statistical analyses were performed using the SPSS9(QUANER) version 16.0 software package (SPSS Inc. Chicago, IL, USA). A paired samples $t$ test was used to analyse the differences between the gastric cancer samples and the paired adjacent noncancerous tissue samples. Associations between LRPPRC expression and clinicopathological characteristics were analyzed by the MannWhitney test and the Kruskal-Wallis test. Survival curves were estimated using the Kaplan-Meyer method, and the log rank test was used to calculate differences between the curves. Prognostic factors were examined by univariate and multivariate analyses (Cox proportional hazards model). A probability level of 0.05 was chosen for statistical significance.

\section{Results}

LRPPRC expression in clinical tissue specimens

LRPPRC expression was investigated by immunohistochemistry in 253 gastric cancer tissues and paired noncancerous tissues. We found that positive LRPPRC expression in gastric cancer tissues $(219 / 253,86.6 \%)$ was significantly higher than that in paired noncancerous tissues $(132 / 253,52.2 \%)$. The difference in LRPPRC staining between gastric cancer tissues and paired noncancerous tissues was statistically significant $(P<0.001)$ (Fig. 1). In 

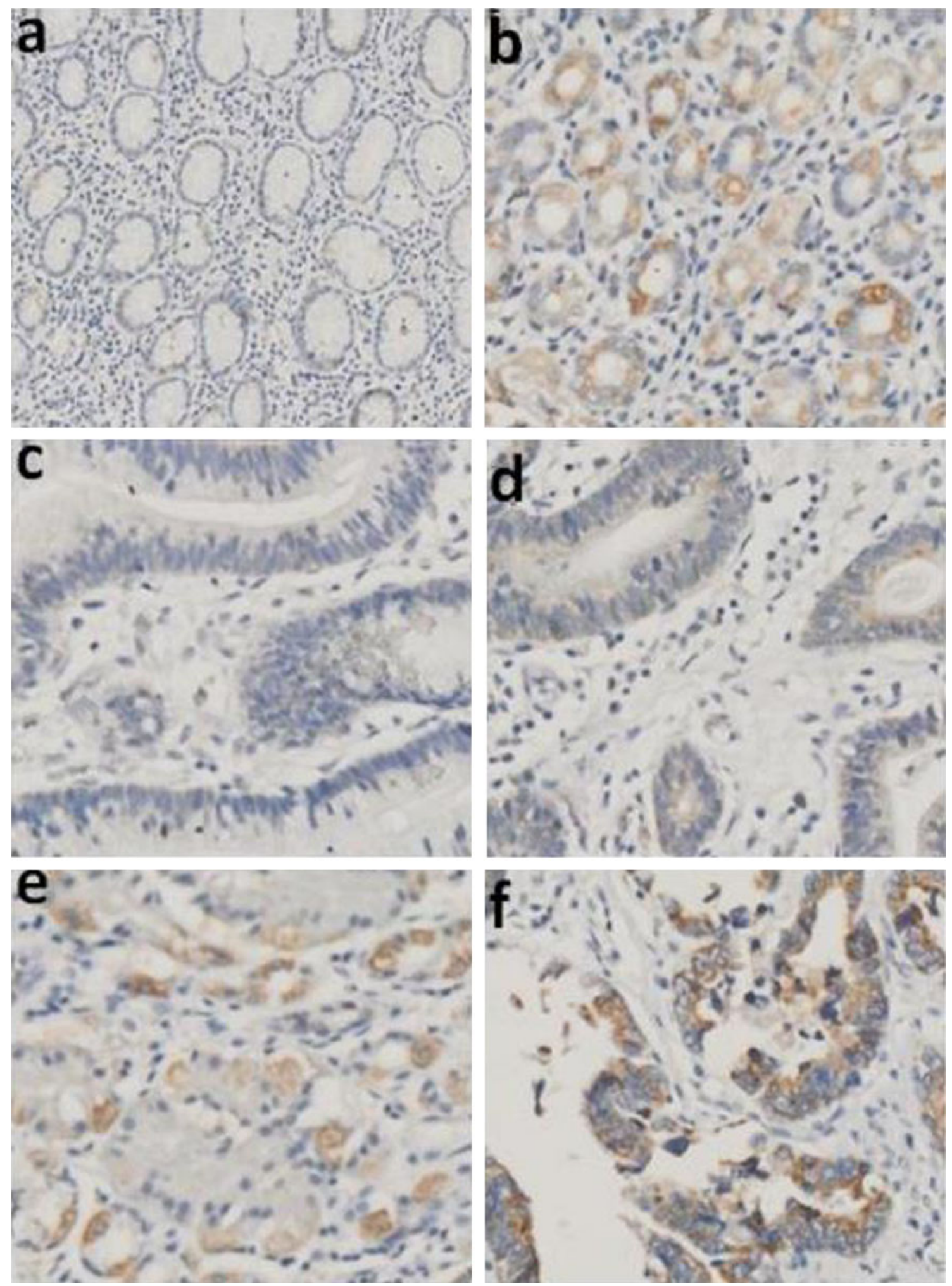

Fig. 1 Immunohistochemical staining of LRPPRC in GC tissues (original magnification, $\times 200$ ).a Negative staining $(-)$ of LRPPRC in normal mucosa. b Moderate staining $(++)$ of LRPPRC in normal mucosa. c Negative staining (-)of LRPPRC in GC tissue. d Weak

staining (+) of LRPPRC in GC tissue. e Moderate staining $(++)$ of LRPPRC in GC tissue. f Strong staining $(+++)$ of LRPPRC in GC tissue 
details, positive immunoreactivity was observed in $86.6 \%$ gastric cancer tissues, with $47(21.4 \%)$ displaying weak $(+)$ positive expression, $83(38 \%)$ moderate $(++)$ positive expression, and $89(40.6 \%)$ strong $(+++)$ positive expression. In contrast, in the paired noncancerous tissues, negative expression of LRPPRC was detected in $47.8 \%$ $(121 / 253)$ of the specimens.

\section{LRPPRC expression and clinicopathological} characteristics

According to LRPPRC expression status, we divided the gastric cancer samples into three groups for the clinicopathological evaluation. The correlation between LRPPRC and clinicopathological features in gastric cancer was further evaluated. The number of cases that were based on histological grade was 34, 138, and 81 in the well, moderate, and poor, respectively. The results showed that increased expression of LRPPRC was significantly correlated with the depth of tumor infiltration ( $\mathrm{T}$ stage, $P<0.001, \mathrm{~N}$ stage $P<0.001$ and $\mathrm{M}$ stage $P=0.002$ ), whereas not with age, gender, tumor locus, tumor grade and TNM stage.

\section{Relationship between LRPPRC expression} and prognosis

The overall survival analysis using the Kaplan-Meyer method revealed that the prognosis of gastric cancer patients whose tumors with higher or moderate LRPPRC expression showed significantly shorter survival than those with no or weak LRPPRC expression ( $P<0.001$; Fig. 2). Table 2 provides the univariate and multivariate analyses

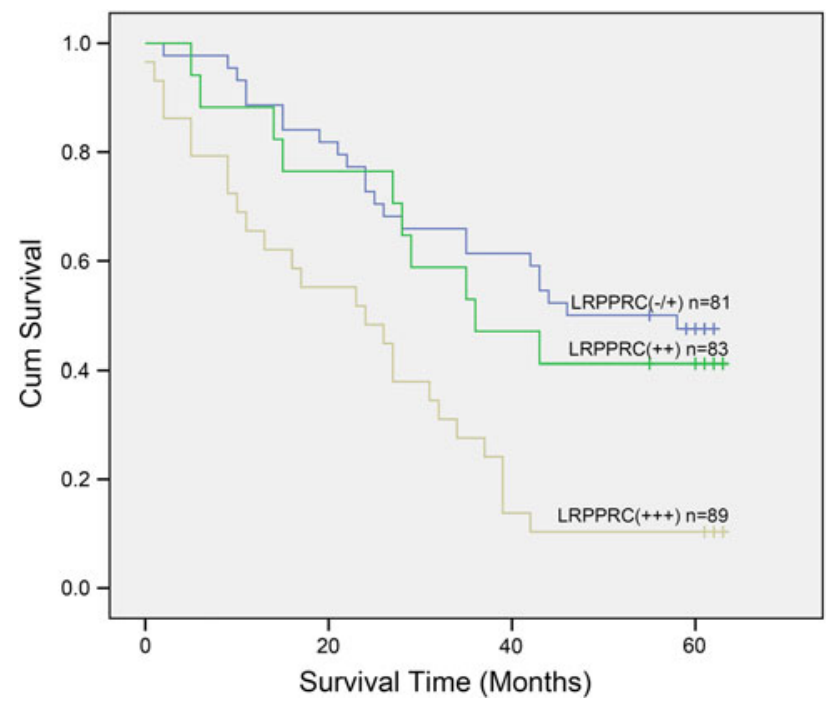

Fig. 2 Kaplan-Meier postoperative survival curve for patterns with gastric cancer and LRPPRC expression of factors related to patient prognosis. Univariate analysis shows that the following factors were significantly related to postoperative survival: depth $(P=0.004)$, lymph node metastasis $(P=0.007)$, distant metastasis $(P<0.001)$, TNM stage $(P=0.004)$, and LRPPRC expression $(P<0.001)$. Furthermore, a Multivariate analysis indicated that lymph node metastasis (N $P=0.004)$, distant metastasis (M $P=0.002)$, TNM stage $(P=0.017)$, and LRPPRC expression $(P=0.004)$ were independent prognostic factors of overall survival for the patients with gastric cancer.

In vitro assessment of LRPPRC expression knockdown

Because LRPPRC expression was higher in gastric cancer tissues than that in paired noncancer tissues, six gastric cancer cell lines were chosen for the proliferation study. We first examined the expression of LRPPRC in six gastric cancer cell lines by Western blot. Our results showed that LRPPRC protein level was higher expressed in gastric cancer cell line SGC7901, BGC823, MKN45, and XGC9811 (Fig. 3a) as compared with the other gastric cancer cell lines. After cell transfection, the expression of LRPPRC in transfected cells was determined by Western blotting. It was found that LRPPRC expression was significantly reduced in SGC7901,BGC823,MKN45, and XGC9811 (Fig. 3b). In proliferation assay, there were differences in cell numbers of SGC7901 between NC and LRPPRC siRNA ( $P<0.05$; Fig. 4). There was no statistically significant difference in the number between the NC and LRPPRC siRNA in the other cell lines.

\section{Discussion}

Gastric cancer is usually a disease of the aged, with the mean patient age ranging between 50 and 70 years. It is thought that gastric cancer results from a combination of environmental factors and an accumulation of generalized and specific genetic alterations [16]. The treatment for gastric cancer includes a combination of surgery, chemotherapy, and radiation therapy. There have been some studies on the prognostic impact of tumor markers in gastric cancer, but the previous studies have not evaluated the relevance in LRPPRC expression and tumor prognosis. The assessment of biological prognostic factors is of clinical importance, especially for a disease with poor outcome such as gastric cancer.

The primary aim of this study was to determine LRPPRC expression and its correlation with clinicopathological, characteristics, and prognosis of patients with gastric cancer. To investigate the potential oncogenic role of LRPPRC in gastric cancer, we first examined the 
Table 2 Univariate and multivariate analyses of overall survival of gastric cancer patients
$H R$ hazard ratio, $C I$ confidence interval, a Numbers of cases in each group; $*$ statistically significant $(P<0.05)$

\begin{tabular}{|c|c|c|c|c|c|c|}
\hline \multirow[t]{2}{*}{ Variables } & \multicolumn{2}{|c|}{ Univariate analyses } & \multirow[t]{2}{*}{$P$} & \multicolumn{2}{|c|}{ Multivariate analyses } & \multirow[t]{2}{*}{$P$} \\
\hline & HR & $(95 \% \mathrm{CI})$ & & HR & $(95 \% \mathrm{CI})$ & \\
\hline Age & & & 0.055 & & & \\
\hline$\leq 65.5$ & 1.000 & & & & & \\
\hline$>65.5$ & 1.658 & $0.990-2.776$ & & & & \\
\hline Gender & & & 0.906 & & & \\
\hline Male & 1.000 & & & & & \\
\hline Female & 1.035 & $0.583-1.838$ & & & & \\
\hline Location & & & 0.909 & & & \\
\hline Gastric antrum & 1.000 & & & & & \\
\hline Lessercurvature & 1.067 & $0.551-2.066$ & & & & \\
\hline Gastric cardia & 1.147 & $0.614-2.145$ & & & & \\
\hline $\mathrm{T}$ & & & $0.004 *$ & & & 0.094 \\
\hline $\mathrm{T} 1$ & 1.000 & & & 1.000 & & \\
\hline $\mathrm{T} 2$ & 1.056 & $0.096-11.643$ & & 1.100 & $0.081-14.873$ & \\
\hline $\mathrm{T} 3$ & 3.913 & $0.537-28.492$ & & 1.603 & $0.201-12.770$ & \\
\hline $\mathrm{T} 4$ & 8.575 & $1.131-64.990$ & & 3.822 & $0.460-31.752$ & \\
\hline $\mathrm{N}$ & & & $0.007 *$ & & & $0.004 *$ \\
\hline N0 & 1.000 & & & 1.000 & & \\
\hline N1 & 1.546 & $0.613-3.899$ & & 1.300 & $0.476-3.550$ & \\
\hline $\mathrm{N} 2$ & 2.104 & $0.943-4.696$ & & 1.343 & $0.393-4.590$ & \\
\hline N3 & 3.601 & $1.653-7.841$ & & 4.635 & $1.321-16.265$ & \\
\hline M & & & $<0.001 *$ & & & $0.002 *$ \\
\hline M0 & 1.000 & & & 1.000 & & \\
\hline M1 & 7.098 & $3.104-16.230$ & & 4.792 & $1.739-13.204$ & \\
\hline TNM & & & $0.004 *$ & & & $0.017 *$ \\
\hline 1 & 1.000 & & & 1.000 & & \\
\hline 2 & 1.957 & $0.562-6.812$ & & 8.991 & $2.208-36.611$ & \\
\hline 3 & 3.120 & $0.745-13.063$ & & 9.819 & $1.878-51.347$ & \\
\hline 4 & 4.892 & $1.500-15.946$ & & 10.847 & $2.057-57.213$ & \\
\hline Tumor grade & & & 0.190 & & & \\
\hline Well & 1.000 & & & & & \\
\hline Moderately & 1.089 & $0.442-2.688$ & & & & \\
\hline Poorly & 1.718 & $0.716-4.124$ & & & & \\
\hline LRPPRC & & & $<0.001 *$ & & & $0.004 *$ \\
\hline \pm & 1.000 & & & 1.000 & & \\
\hline++ & 1.230 & $0.585-2.585$ & & 1.060 & $0.475-2.365$ & \\
\hline+++ & 3.012 & $1.698-5.341$ & & 2.883 & $1.472-5.647$ & \\
\hline
\end{tabular}

expression level of LRPPRC in a series of paired gastric cancer tissues with the adjacent nonneoplastic tissues. The expression of LRPPRC was verified by immunohistochemistry in gastric cancer and corresponding normal tissues. Results showed that the LRPPRC expression levels were significantly increased in tumor tissue samples, compared with that in the adjacent nontumor tissue samples, as illustrated in Fig. 1. The similar result was obtained in other studies showing that LRPPRC protein was indeed relatively upregulated in gastric cancer and others carcinoma tissues. ${ }^{16}$ In addition, according to analysis of the correlation between expression level of LRPPRC and patients' characteristics, one of the interesting findings was that expression level of LRPPRC was significantly associated with the depth of tumor invasion, lymph nodes metastasis ( $\mathrm{N}$ stage), and distant metastasis (M stage) (Table 1).Tian et al. [11] reported that the lung adenocarcinoma cell line A549, treated with LRPPRC, had high invasive ability. The present in vitro study showed that LRPPRC expression is associated with tumor growth, and 
A

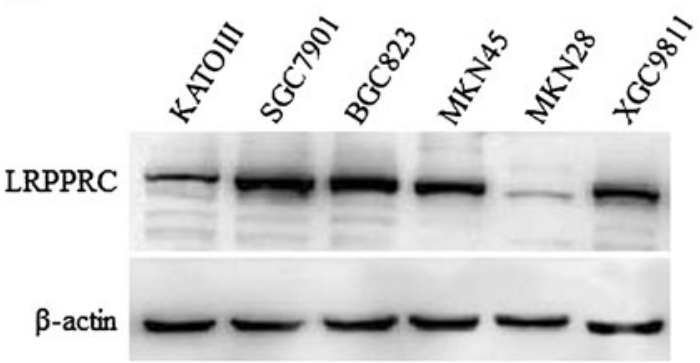

Fig. 3 a Expression of LRPPRC in the gastric cancer cell lines. $\beta$ actin was used as an internal control. The level of LRPPRC protein expression was significantly higher in the gastric cancer cell line

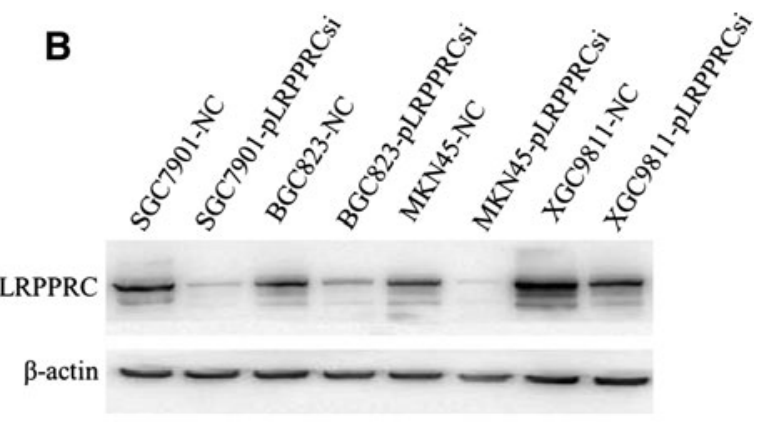

SGC7901, BGC823, MKN45, and XGC9811 than in other cell lines. b Expression of LRPPRC siRNA in transfected cells detected by Western blot analysis
Fig. 4 Proliferation assay and siRNA inhibition in 4 gastric cancer cell lines. The proliferation assay showed a difference in growth of gastric cancer cell line SGC7901.

There were significant differences between $\mathrm{NC}$ and LRPPRC siRNA. In the other 3 cell lines, there was no significant difference between NC and LRPPRC siRNA (a SGC7901; b BGC823; c MKN45; d XGC9811). Values are mean \pm SD for three independent experiments. $N C$ negative control
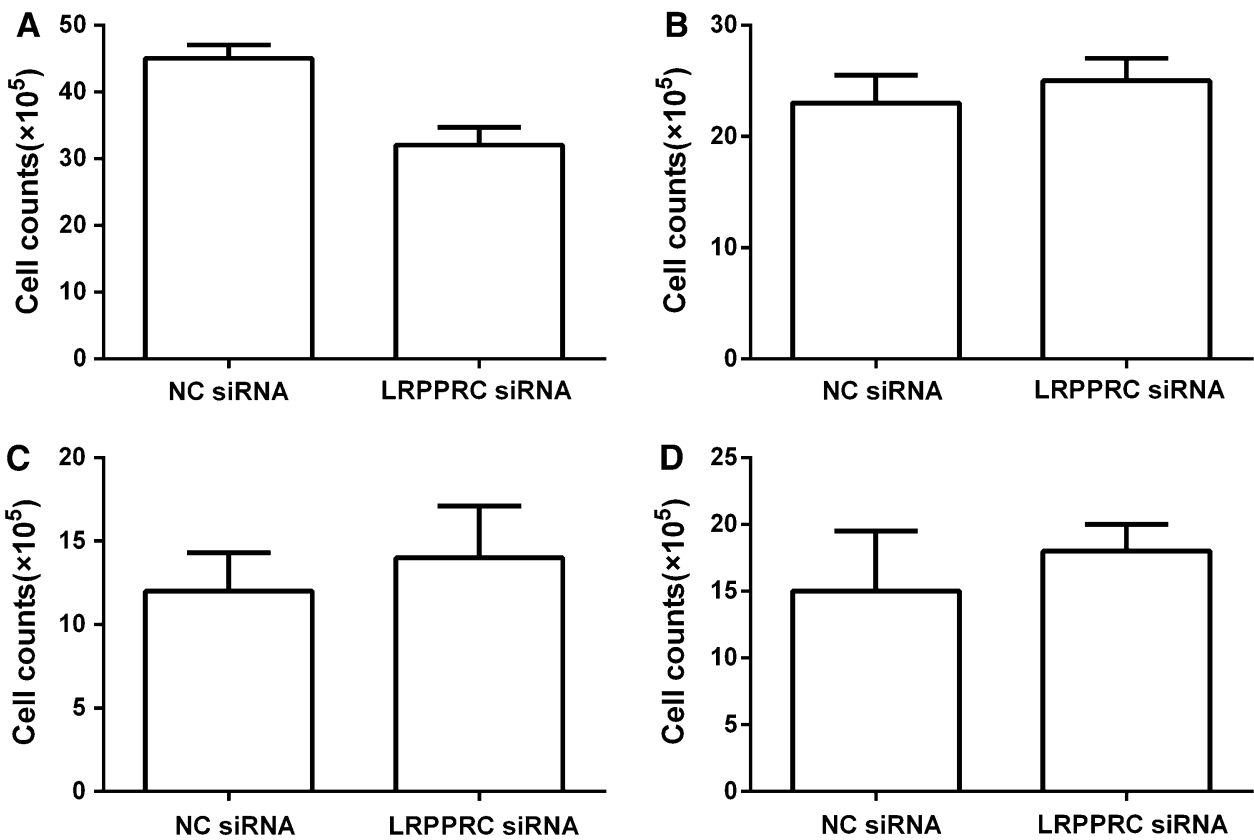

the inhibition of LRPPRC may lead to a reduction in gastric cancer proliferation. These results suggest that LRPPRC may play an important role in the tumorigenesis of gastric cancer (Fig. 4).

One of the most important findings in this study was that high expression of LRPPRC in gastric cancer patients was significantly associated with poor prognosis and low overall survival, as shown in Fig. 2, indicating that high LRPPRC protein level is a marker of poor prognosis for patients with gastric cancer. Multivariate analyses in Table 2 further revealed that only lymph node metastases and distant metastases, TNM stage, and expression of LRPPRC were independent prognostic factors in patients with gastric cancer.

In summary, our results show that high LRPPRC protein expression is correlated with the depth of tumor infiltration and is an unfavorable independent prognostic factor for surgically resected gastric cancer. The metastasis mechanism of LRPPRC in gastric cancer may involve a multitude of epigenetic pathways and needs to be addressed in the future. These findings suggest that LRPPRC can serve as a predictive marker of patient outcome in gastric cancer. Since the number of patients in this study was small, further study of a larger patient population is necessary to confirm its clinical significance in gastric cancer.

Acknowledgments Supported by the National Natural Science Foundation of China (No. 30971134, No. 81272344, No.81000890, No. 81372169) and the National Key and Basic Research Development Program of China (No. 2010CB529300, 05, 06). We would like to thank Mr.Li for critical reading of the manuscript.

Conflict of interest The authors declare that they have no competing interests.

Open Access This article is distributed under the terms of the Creative Commons Attribution License which permits any use, 
distribution, and reproduction in any medium, provided the original author(s) and the source are credited.

\section{References}

1. Jemal A, Siegel R, Ward E, Hao Y, Xu J, et al. Cancer statistics, 2008. CA Cancer J Clin. 2008;58:71-96.

2. Hartgrink HH, Jansen EP, van Grieken NC, van de Velde CJ. Gastric cancer. Lancet. 2009;374:477-90.

3. Parkin DM, Bray FI, Devesa SS. Cancer burden in the year 2000. The global picture. Eur J Cancer. 2001;37(Suppl 8):S4-66.

4. Santoro R, Carboni F, Lepiane P, Ettorre GM, Santoro E. Clinicopathological features and prognosis of gastric cancer in young European adults. Br J Surg. 2007;94:737-42.

5. Zhao ZS, Wang YY, Chu YQ, et al. SPARC is associated with gastric cancer progression and poor survival of patients. Clin Cancer Res. 2010;16(1):260-8.

6. Small ID, Peeters N. The PPR motif: a TPR-related motif prevalent in plant organellar proteins. Trends Biochem Sci. 2000;25:46-7.

7. Barkan A, Rojas M, Fujii S, Yap A, Chong YS, Bond CS, Small I. A combinatorial amino acid code for RNA recognition by pentatricopeptide repeat proteins. PLoS Genet. 2012;8:e1002910.

8. Xu F, Morin C, Mitchell G, Ackerley C, Robinson BH. The role of the LRPPRC (leucine-rich pentatricopeptide repeat cassette) gene in cytochrome oxidase assembly: mutation causes lowered levels of COX (cytochrome c oxidase) I and COX III mRNA. Biochem J. 2004;382:331e6.

9. Sasarman F, Brunel-Guitton C, Antonicka H, Wai T, Shoubridge EA. Consortium L.LRPPRC and SLIRP interact in a ribonucleoprotein complex that regulates posttranscriptional gene expression in mitochondria. Mol Biol Cell. 2010;21:1315e23.

10. Cooper MP, Qu L, Rohas LM, et al. Defects in energy homeostasis in Leigh syndrome French Canadian variant through PGC1alpha/LRP130 complex. Genes Dev. 2006;20:2996-3009.

11. Tian Tian, Ikeda Jun-ichiro, Wang Yi, et al. Role of leucine-rich pentatricopeptide repeat motif-containing protein (LRPPRC) for anti-apoptosis and tumourigenesis in cancers. Eur $\mathrm{J}$ Cancer. 2012;48:2462-73.

12. Li X, Hao Z, Fan R, Zou X, Jin H, Pan Y, He L, Du R, Gao L, Liu D. Fan D:CIAPIN1 inhibits gastric cancer cell proliferation and cell cycle progression by downregulating CyclinD1 and upregulating P27. Cancer Biol Ther. 2007;6(10):1539-45.

13. Hao Z, Li X, Qiao T, Li S, Lv Y, Fan D. Downregulated expression of CIAPIN1 may contribute to gastric carcinogenesis by accelerating cell proliferation and promoting cell cycle progression. Cancer Biol Ther. 2009;8(11):1064-70.

14. He L, Wang H, Jin H, Guo C, Xie H, Yan K, Li X, Shen Q, Qiao T, Chen G, Chai N, Zhao L, Dong Q, Zheng Y, Liu J. Fan D:CIAPIN1 inhibits the growth and proliferation of clear cell renal cell carcinoma. Cancer Lett. 2009;276(1):88-94.

15. Shi H, Chen S, Jin H, Xu C, Dong G, Zhao Q, Wang W, Zhang H, Lin W, Zhang J, Davidovic L, Yao L, Fan D. Downregulation of MSP58 inhibits growth of human colorectal cancer cells via regulation of the cyclin D1-cyclin-dependent kinase 4-p21 pathway. Cancer Sci. 2009;100(9):1585-90.

16. Tavares A, Gandra A, Viveiros F, Cidade C, Maciel J. Analysis of clinicopathologic characteristics and prognosis of gastric cancer in young and older patients. Pathol Oncol Res. 2013;19:111-7. 